\title{
A Novel Hybrid Bi-orthogonal Wavelets/ADPCM Algorithm For Very Low Bit Rate Satellite Image Compression
}

\author{
Tao Yu, Anthony Tung-Shuen Ho, Siu-Chung Tam, \\ Siong-Chai Tan and Lian-Teck Yap \\ Nanyang Technological University, \\ School of Electrical and Electronic Engineering, Nanyang Avenue, Singapore 639798 \\ Tel: (65)-790-5466 / Fax: (65)-792-0415 / E-mail: etyu@ntu.edu.sg
}

\begin{abstract}
In this paper, we present an overview of remote sensing image compression using wavelet transforms. We begin by describing the wavelet properties that are most important for image compression. In particular, we present a method to construct bi-orthogonal wavelets and their Finite Impulse Response (FIR) filter banks. All of these FIR filter banks all have linear phase characteristics and the signal can be reconstructed exactly. Next, we expound on a novel hybrid scheme that uses bi-orthogonal wavelets and the adaptive differential pulse code modulation (ADPCM) algorithm for very low bit-rate satellite image compression.
\end{abstract}

Complicated SPOT images of city scenes that contain many high frequency details, such as building structures and roads, are used in this investigation. Using our hybrid wavelet/ADPCM compression algorithm, the data integrity of the satellite image was maintained with a Peak Signal-to-Noise Ratio (PSNR) of approximately 26dB while achieving a compression ratio of 150:1.

\section{INTRODUCTION}

Due to the vast amount of digital images associated with satellite remote sensing, image compression has become a key technology for the transmission and storage of satellite images. In the past ten years, the spatial and spectral resolutions of satellite remote sensing have increased significantly. The need for more efficient data compression schemes is even more apparent. This in turn has attracted growing interest from the remote sensing community.

Satellite images contain typically much less redundancy information than conventional natural images. It is therefore not an easy task to achieve high compression ratios for these images. For natural images, good compression ratios can be achieved through ADPCM. However, for satellite images that contain high frequency details, ADPCM achieved adequate performance only at low compression ratios. Other techniques, such as vector quantization (VQ), have found numerous applications in speech and image signal processing. But the blocking effects of VQ have restricted the application of VQ codes in satellite image compression.

The topic of "wavelet analysis" has recently attracted much attention from both mathematicians and engineers. Wavelet theory has found applications in areas such as image analysis, communication systems, biomedical imaging, radar, and control systems. However, many literatures associated with wavelets require that the reader has a good knowledge in both functional analysis and group representation theory. The application of bi-orthogonal wavelets to construct linear phase FIR filter pairs is presented in this paper. Using these FIR filters, the satellite image is first transformed into the time-frequency domain. A combination of VQ and ADPCM algorithms is then used to achieve very low bit-rate compression. Image reconstruction is performed using the inverse wavelet transform. As VQ performs image coding in different sub-bands, blocking efforts in satellite images are also minimized.

\section{CONSTRUCTION OF BI-ORTHOGONAL WAVELET BASIS AND ITS FIR FILTER BANK}

Wavelet transform is a new 2-D time-frequency signal analysis method. Haar described the first wavelet basis back in 1910. In the $1980 \mathrm{~s}$, many researchers [1] performed pioneering work into wavelets, particularly in multiresolution and fast wavelet transforms resulting in numerous signal processing applications.

The frame of multi-resolution analysis is to generate a closed subspaces $\left\{V_{m}\right\}$ and $\left\{W_{m}\right\}$ in the $L^{2}(R)$ space. The functions $\phi_{m, n}(t)$ are the regular orthogonal basis of the subspace $\left\{V_{m}\right\}$ and wavelets $\varphi_{m, n}(t)$ are used to construct the subspace $\left\{W_{m}\right\}$, and $m \in Z$. They have the following properties:

$$
V_{m} \perp W_{m} \quad V_{m+1}=V_{m}+W_{m}
$$

There exists two sequences $\left\{p_{k}\right\}$ and $\left\{q_{k}\right\} \in \ell^{2}$ such that

$$
\left\{\begin{array}{l}
\phi(x)=\sum_{k} p_{k} \phi(2 x-k) \\
\varphi(x)=\sum_{k} q_{k} \phi(2 x-k)
\end{array}\right.
$$

Normally, we call the unique sequence $\left\{p_{n}\right\}$ that relates $\phi(x)$ with the functions $\phi(2 x-k), k \in Z$ "two-scale function", its Z-transform is $H(z)$. For an orthogonal wavelet transform, $H(z)$ satisfies

$$
|H(z)|^{2}+|H(-z)|^{2}=1 \text {, where }|z|=1 \text {. }
$$


Although orthogonal analysis is very good in signal processing, The basis of orthogonal wavelet transform is generally neither compact nor continuous. That is, it is not easy to design linear FIR filters based on orthogonal wavelets. In order to apply a wavelet transform in image processing requires linear phase FIR filters. In this paper, biorthogonal wavelets are considered. $\mathrm{Bi}$-orthogonal wavelets mean that two multi-resolution analyses $\left\{V_{m}\right\},\left\{W_{m}\right\}$ and $\left\{\tilde{V}_{m}\right\},\left\{\tilde{W}_{m}\right\}$ satisfy the following condition:

$$
V_{m} \perp \tilde{W}_{m}, \widetilde{V}_{m} \perp W_{m}
$$

This means that their Z-transforms satisfy ${ }^{[2]}$ :

$$
|H(z)\|\tilde{H}(z)|+| H(-z)\| \tilde{H}(-z)|=1 \text {, where }|z|=1 \text {. }
$$

During the design of a wavelet filter, several important points must be taken into consideration. Among them are: length of the filter, computational complexity and efficiency, simplicity in implementation, smoothness and symmetry of the basic wavelet, and the order of approximation.

In our approach, we design bi-orthogonal wavelet filters with B-splines. B-splines are multi-resolution scaling functions and the support is compact. In general, B-splines could generate a multi-resolution basis of $L^{2}(R)$, and consequently the B-splines would satisfy the two-scale relationship ${ }^{[2]}$ :

$$
N_{m}(t)=\sqrt{2} \sum_{n=0}^{N} p_{m, n} N_{m}(2 x-n)
$$

(where $N_{m}(t)$ is a B-spline function)

Its Fourier transform is:

$$
\hat{N}_{m}(2 \omega)=H(\omega) \cdot \hat{N}_{m}(\omega)
$$

Therefore, for $\mathrm{m}=2, p_{2, n}$ could be obtained using the inverse Fourier transform:

$$
p_{2,0}=\sqrt{2} / 4, p_{2,1}=\sqrt{2} / 2, p_{2,2}=\sqrt{2} / 4
$$

After we get the $H(\omega), \widetilde{H}(\omega)$ can be obtained using:

$$
\begin{aligned}
& H(\omega) \cdot \widetilde{H}(-\omega)= \\
& {\left[\cos ^{2}\left(\frac{\omega}{2}\right)\right]^{N}\left\{\begin{array}{l}
\sum_{k=0}^{N-1}\left(\begin{array}{c}
N-2+k \\
k
\end{array}\right) \sin ^{2 k}\left(\frac{\omega}{2}\right)+ \\
\sin ^{2 N}\left(\frac{\omega}{2}\right) \cdot R\left(\frac{1}{2} \cos w\right)
\end{array}\right\}}
\end{aligned}
$$

Substituting $\mathrm{N}=3, R(\omega)=0=0$ and $H(\omega)$,

$$
\widetilde{H}(-\omega)=\left(\frac{1+\cos \omega}{2}\right)^{2}\left[1+3 \frac{1-\cos \omega}{2}+6\left(\frac{1-\cos \omega}{2}\right)^{2}\right]
$$

Then, the wavelet transform FIR filter pairs can be obtained as shown in Table 1: $\left(m=2, m^{\prime}=4\right)$. Figl shows $|H(\omega)|$ versus $\omega$. Similarly, other FIR filter pairs can be obtained from Tables 2. All these filter banks have the following important characteristics:

- They satisfy the bi-wavelet analysis conditions.

- Their bases are compact support and continuous.

- High efficiency.

- Linear phase.

- Exact reconstruction of signal.

These characteristics are very useful in transforming an image to time-frequency domain. Now, let us employ them into satellite image compression.

Table1: Bi-orthogonal Wavelet FIR Filter Pair $\left(m=2, m^{\prime}=4\right)$

\begin{tabular}{|c|c|c|c|c|c|}
\hline $\mathrm{n}$ & 0 & \pm 1 & \pm 2 & \pm 3 & \pm 4 \\
\hline$p_{n}$ & $\sqrt{2} / 2$ & $\sqrt{2} / 4$ & 0 & 0 & 0 \\
\hline$\widetilde{p}_{n}$ & $45 \sqrt{2} / 64$ & $16 \sqrt{2} / 64$ & $-\sqrt{2} / 8$ & $-3 \sqrt{2} / 64$ & $3 \sqrt{2} / 128$ \\
\hline
\end{tabular}

Table1: Bi-orthogonal Wavelet FIR Filter Pair $\left(m=2, m^{\prime}=2\right)$

\begin{tabular}{|c|c|c|c|c|c|}
\hline $\mathrm{n}$ & 0 & 1 & 2 & 3 & 4 \\
\hline$p_{n}$ & $\sqrt{2} / 4$ & $\sqrt{2} / 2$ & $\sqrt{2} / 4$ & 0 & 0 \\
\hline$\widetilde{p}_{n}$ & $-3 \sqrt{2} / 16$ & $\sqrt{2} / 4$ & $7 \sqrt{2} / 8$ & $\sqrt{2} / 4$ & $-3 \sqrt{2} / 16$ \\
\hline
\end{tabular}

\section{SATELLITE IMAGE COMPRESSION}

The objective of digital image compression is to minimize the number of bits required to represent an image, while maintaining acceptable image quality.

The statistics of an image will change after a wavelet transformation. Applying a low-pass wavelet filter several times would result in the coarse components becoming highly correlated and ideally suited for ADPCM. However, the pixel values associated with the detail components of the image would suffer from random reduction of magnitude. Therefore, another method is needed to compress these high frequency components.

Vector Quantization is a good method to compress images to very low bit rates. The blocking effects of a VQ decoded image, however, limits the application of VQ coding in images, especially in satellite image compression. There are two methods to improve the overall image quality with VQ coding: one is to optimize the quality of the designed codebooks; the other is to improve the data statistics for vector quantization.

Satellite images have typically different statistical parameters; it is almost impossible to get common codebooks to satisfy all of them. DCT and FFT are two methods that can be used to convert images from the spatial domain to the frequency domain to improve their statistical characteristics, but both of them could not keep any spatial information (unless sub-block is used, but it will cause the blocking effect). 
Wavelet image analysis offers the opportunity to compress the images at different scales and subbands. In this paper, the bi-orthogonal wavelet FIR filter is used in image transform. In the initial iteration of the bi-orthogonal wavelet FIR filter algorithm, the remote sensing image data is decomposed into one coarse and three detail components. In the next iteration stage the obtained coarse component of the previous iteration is decomposed into further coarse and detail components. So that after $\mathrm{N}$ decomposition iterations, there are $3 \mathrm{~N}$ detail components and one coarse component of the original image. The coarse component of the decomposition can be understood as a low-pass filtered subimage and the detail components as the high-pass filtered sub-images of the original signal.

The basic idea of our novel hybrid Bi-orthogonal Wavelets/ADPCM algorithm can be described as follow: Wavelet transform changes the statistics of data. After several times of low-pass filter, the coarse component becomes highly correlated and is suitable for ADPCM coding. The detail components are quite different. The pixel values in these components are always small and poorly correlated. They are not suitable for ADPCM and Vector Quantization is used to compress the data.

\section{EXPERIMENTAL RESULTS}

In this section, we present some experimental results of satellite image compression by applying the Hybrid Biorthogonal Wavelet Transform/ADPCM Algorithm.

We use $m=2$ and $m^{\prime}=4$ bi-orthogonal wavelet FIR filter pairs to transform the image into the time-frequency domain. After three wavelet transforms, nine detail components and one coarse component are obtained. Then a (8:3) ADPCM compression algorithm is used for the coarse component

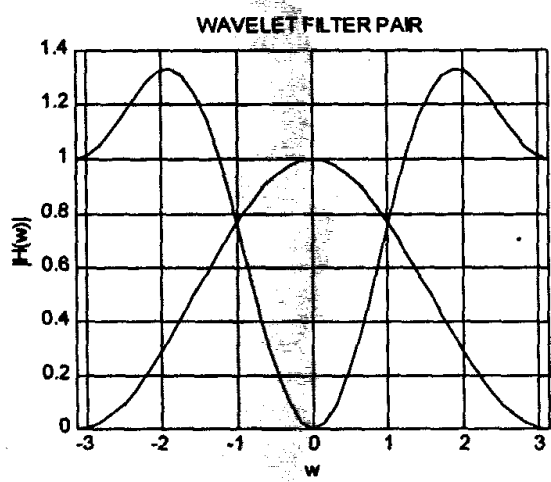

Fig1. Wavelet Filter Pair

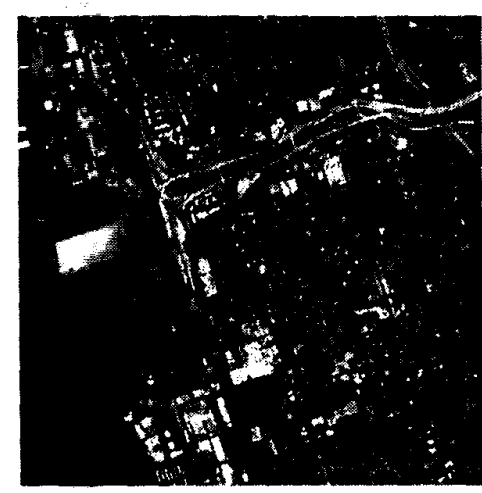

a.) Original Image

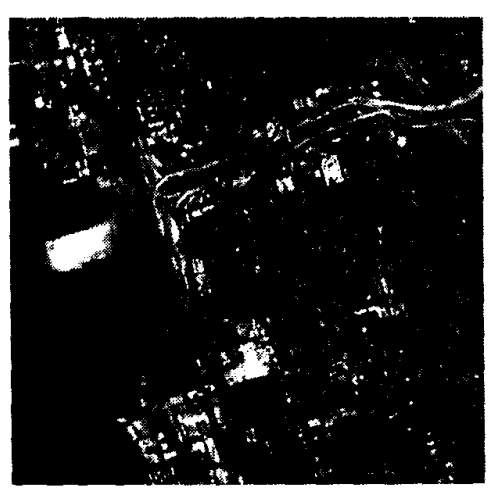

b.) Reconstructed Image (Compression ratio $=150: 1$ PSNR $=25.88 \mathrm{~dB}$
Fig2. Remote Sensing Image Compression with Novel Hybrid Bi-orthogonal Wavelets/ADPCM Algorithm 